\title{
Forecasting the South African Inflation Rate: On Asymmetric Loss and Forecast Rationality
}

\author{
November 2014
}

\begin{abstract}
Using forecasts of the inflation rate in South Africa, we study the rationality of forecasts and the shape of forecasters' loss function. When we study micro-level data of individual forecasts, we find mixed evidence of an asymmetric loss function, suggesting that inflation forecasters are heterogeneous with respect to the shape of their loss function. We also find strong evidence that inflation forecasts are in line with forecast rationality. When we pool the data, and study sectoral inflation forecasts of financial analysts, trade unions, and the business sector, we find evidence for asymmetry in the loss function, and against forecast rationality. Upon comparing the micro-level results with those for pooled and sectoral data, we conclude that forecast rationality should be assessed based on micro-level data, and that freer access to this data would allow more rigorous analysis and discussion of the information content of the surveys.
\end{abstract}

JEL classification: C53, D82, E37

Keywords: Inflation rate; Forecasting; Loss function; Rationality

\section{Addresses:}

* Christian Pierdzioch, Helmut-Schmidt-University, Department of Economics, Holstenhofweg 85, P.O.B. 700822, 22008 Hamburg, Germany. Email: c.pierdzioch@hsu-hh.de.

Monique B. Reid. Stellenbosch University, Department of Economics, Private Bag X1, Matieland, South Africa, 7602. Email: mreid@sun.ac.za.

Rangan Gupta, Department of Economics, University of Pretoria, Pretoria, 0002, South Africa. Email: rangan.gupta@up.ac.za.

* Corresponding author. 


\section{Introduction}

The question of how economic agents form expectations about inflation is crucially important to monetary policymakers as well as anyone who tracks inflation as an indicator of macroeconomic performance in an economy. We study how economic agents form inflation expectations in South Africa. Individually and as part of groups such as BRICS, South Africa and other emerging economies are playing an increasing role in the global economy and it is not obvious that understanding trends in major industrial countries is sufficient to explain those experienced in emerging markets. There is ongoing research (Kose and Prasad 2010) about the extent of the sensitivity of emerging markets to shocks originating in industrial countries and whether the business cycles of emerging market countries are converging with or diverging (decoupling) from those of the advanced economies. South Africa's economy and financial sector in particular plays a dominant in the African context as well as via its economic links with influential emerging market countries such as China and India.

Kose and Prasad (2010) argue that the rising prominence of emerging market economies can to a large extent be attributed to improved policy frameworks and institutions, as well as the ability to implement counter-cyclical economic policies effectively. One of the first indications of the macroeconomic performance of a country is the health of the monetary policy of that country. Measuring the health of the monetary policy of a country, in turn, requires a careful analysis of the dynamics of inflation and of inflation expectations in particular. Our research on the formation of inflation expectations, therefore, is valuable in that it extends our understanding of the monetary policy environment in a potentially strategically important emerging market economy.

Part of the literature attempting to understand the formation of inflation expectations has focused on various facets of the question of whether inflation expectations are rational (Figlewski and Wachtel 1981, Rich 1989, Keane and Runkle 1990, Roberts 1997, Nunes 2010, among others). From a monetary policy perspective, the assumption of full rationality would allow only unexpected changes in inflation to affect real variables. For South Africa, Ehlers and Steinbach (2007) have undertaken a comprehensive analysis of the rationality of inflation expectations using the Bureau for Economic Research (BER) inflation expectations survey data and data from the Reuters Inflation Expectations (RIE) Survey. They find that the inflation expectations of financial analysts and short-term expectations by the trade unions appear unbiased. In contrast, the expectations of forecasters from the business sector and the longer-term expectations of trade unions seem to be biased. Moreover, expectations from all three groups of forecasters (financial analysts and forecasters from the business sector and trade unions) were found to violate the condition of weak rationality, that is, forecasters seem to use information inefficiently. The forecasts 
for the current-quarter horizon from the RIE Survey are an exception. Similarly, Kabundi and Schaling (2013) also study the BER data and find that inflation expectations are not in line with the rational expectations hypothesis. Specifically, they report that inflation expectations are tied to the lagged inflation rate. In a follow-up study, Kabundi et al. (2014) find that forecasts of price setters (business representatives and trade unions) are linked to the lagged inflation rate, but this holds only to a lesser extent for the forecasts of financial analysts.

Our contribution to the literature on the formation of inflation expectations in South Africa is that we test forecast rationality under the assumption of an asymmetric loss function. In the earlier literature, discussed above, researchers have tested rationality of inflation forecasts by implicitly assuming that forecasters have a quadratic loss function. It is, however, a priori unclear why forecast errors with different signs should lead to loss of an equal magnitude. It is quite reasonable to imagine that in many cases particular agents do not view an overprediction and underprediction as equivalent. For example, the literature suggests that financial analysts/professional economists face a trade-off between two competing incentives - an incentive to forecast accurately and an incentive to strategically bias forecast in some manner (Laster et al. 1999). A range of hypotheses have been proposed to explain these strategic reasons for bias (for a brief summary, see Reid and Du Plessis 2011). Crucially, it is likely that different analysts in the group surveyed will face different incentives, depending on the industry that they work in, giving rise to a certain degree of forecaster heterogeneity. Forecaster heterogeneity, in turn, implies that it is not clear ex ante that unbiased forecasts or overpredictions (underpredictions) should be expected from a given group on average. In fact, as Keane and Runkle (1990) point out, forecaster heterogeneity can have two alternative consequences for tests of forecast rationality. First, while, at the micro level, forecasts from individual forecasters can be perfectly consistent with forecast rationality, there could be strong evidence against rationality of forecasts at the aggregate or sectoral level. Second, aggregation may wash out individual biases of forecasts resulting in spurious evidence of forecast rationality. Hence, we study the shape of forecasters' loss function and the rationality of forecasts using both micro level data on forecasts from individual forecasters and, based on the BER data studied extensively in earlier research, sectoral inflation forecasts.

Elliot et al. $(2005,2008)$ and Patton and Timmermann (2007) point out that relaxing the assumption of symmetric loss has the important consequence that rationality no longer requires the forecast errors to be unbiased. They present a more general framework for rationality testing, which applies to a flexible family of loss functions and even to situations in which the functional form of the loss function is unknown. While testing for forecast rationality under asymmetric loss has become popular in recent years (for an analysis of government forecasts, see Christodoulakis and Mamatzakis 2008; for an analysis of business cycle forecasts, see Döpke et al. 2010; for an 
analysis of forecasts of exchange rates, see Fritsche et al. 2014; for an analysis of central bank forecasts, see Pierdzioch et al. 2014a), most of this research focuses on industrial economies, whereas we look at South African inflation expectations as an example of an emerging market economy.

Our empirical analysis consists of two parts. In the first part, we study micro-level data of individual inflation forecast. We report mixed evidence of an asymmetric loss function. Our results suggest that inflation forecasters are heterogeneous to some extent with respect to the shape of their loss function, but also that symmetry of the loss function cannot be rejected for many forecasters. Importantly, we find strong evidence that inflation forecasts are in line with forecast rationality. Because our micro-level data comprises of the inflation forecasts of financial analysts/professional economists, a natural question is whether the results extend to other groups of forecasters. Hence, in the second part, we pool the micro-level data and compare the results for the pooled data with the results for the sectoral BER data. The BER data comprises of, at a sectoral level, inflation forecasts of financial analysts, trade unions, and the business sector. For the sectoral data, we find evidence in favor of asymmetry of the loss function, and against forecast rationality. As expected, given the micro-level results, the asymmetry of the loss function is less pronounced in the case of financial analysts than for the other two groups of forecasters, which in turn, is consistent with heterogeneity of forecast formation across the three groups of forecasters (Ehlers and Steinbach 2007, Reid and Du Plessis 2011, Reid 2012, Kabundi and Schaling 2014). However, all the South African research papers listed here that use the BER survey data study the aggregate data for each of the three groups which ignores any heterogeneity within these groups of forecasters. Our results suggest that studying aggregate data may not suffice to fully characterize the rationality of inflation forecasts. When we compare both parts of our empirical analysis, we conclude that forecast rationality should be assessed based on micro-level data, and that freer access to such micro-level data on the inflation forecasts of the trade unions and the business sector would allow more rigorous analysis and discussion of the information content of the surveys. This suggestion for South African inflation expectations is in line with other research that documents the advantages of using micro-level data for testing forecast rationality (Keane and Runkle, 1990). A micro-level study of the forecasts of trade unions and the business sector would be particularly important for monetary policy decisions, especially in an inflation-targeting economy like South Africa, because trade unions and the business sector are "price setters" and, hence, their forecast behavior is likely to translate into wage negotiations and eventually inflation dynamics. Reid and du Plessis (2011) and Reid (2012) introduce the term "price setters" when working with the BER survey data, to make this distinction between the relatively more informed financial analysts and the general public who, though they are less informed, are crucial to the understanding of inflation dynamics in South Africa.

We organize the remainder of tha paper as follows: In Section 2, we briefly describe the empirical 
methods that we use to study the shape of forecasters' loss function and the rationality of their forecasts. In Section 3, we describe the two parts of our empirical analysis. In the first part, we describe the micro-level analysis. In the second part, we describe the analysis of pooled and sectoral data. In Section 4, we summarize our main results and offer some concluding remarks.

\section{Empirical Methods}

The approach developed by Elliott et al. (2005) to study the rationality of forecasts under an asymmetric loss function starts with the assumption that forecasters' loss function, $\mathcal{L}$, belongs to the following known functional form:

$$
\mathcal{L}=\left[\alpha+(1-2 \alpha) I_{t+1}\right]\left|\pi_{t+1}-\hat{\pi}_{t \mid t+1}\right|^{p}
$$

where $\pi_{t+1}$ denotes the realization of the inflation rate, $\hat{\pi}_{t \mid t+1}$, denotes the forecast formed in period $t$ of the inflation rate in period $t+1, I_{t+1}=1_{\left\{\pi_{t+1}<\hat{\pi}_{t \mid t+1}\right\}}$ denotes the indicator function, $p=1$ for a lin-lin loss function, and $p=2$ for a quad-quad loss function. While both functional forms assume a minimum at a forecast error of zero, the lin-lin loss function is a piecewise linear function that linearly increases in positive and negative forecast errors. The quad-quad loss function, in turn, is quadratic in the forecast error, where the specific shape of both functions depends on the parameter, $\alpha$. The parameter $\alpha$ can assume values between zero and one. If we set $\alpha=0.5$, we get a symmetric loss function. A quadratic (piecewise linear) symmetric loss function obtains for the parameter configuration $\alpha=0.5$ and $p=2(\alpha=0.5$ and $p=1)$. If we set $\alpha>0.5$ $(\alpha<0.5)$, then underestimation (overestimation) of the inflation rate produces a larger loss than an overestimation (underestimation) of the same size. Estimation of the parameter $\alpha$ can be done using results derived by Elliott et al. (2005). We have

$$
\hat{\alpha}=\frac{\left[\frac{1}{T} \sum_{t=\tau}^{T+\tau-1} v_{t}\left|\pi_{t+1}-\hat{\pi}_{t \mid t+1}\right|^{p-1}\right]^{\prime} \hat{S}^{-1}\left[\frac{1}{T} \sum_{t=\tau}^{T+\tau-1} v_{t} I_{t+1}\left|\pi_{t+1}-\hat{\pi}_{t \mid t+1}\right|^{p-1}\right]}{\left[\frac{1}{T} \sum_{t=\tau}^{T+\tau-1} v_{t}\left|\pi_{t+1}-\hat{\pi}_{t \mid t+1}\right|^{p-1}\right]^{\prime} \hat{S}^{-1}\left[\frac{1}{T} \sum_{t=\tau}^{T+\tau-1} v_{t}\left|\pi_{t+1}-\hat{\pi}_{t \mid t+1}\right|^{p-1}\right]},
$$

where $\hat{\alpha}$ denotes the estimated asymmetry parameter, $\hat{S}=\frac{1}{T} \sum_{t=\tau}^{T+\tau-1} v_{t} v_{t}^{\prime}\left(I_{t+1}-\hat{\alpha}\right)^{2} \mid \pi_{t+1}-$ $\left.\hat{\pi}_{t \mid t+1}\right|^{2 p-2}$, and $v_{t}$ denotes a vector of instruments, $T$ denotes the number of forecasts available, starting at $\tau+1$. Because $\hat{\alpha}$ shows up on both sides of Equation (2), we choose an iterative estimation procedure that stops when the change in $\hat{\alpha}$ is sufficiently small. Once the iteration has stopped, we test the hypothesis that the estimated asymmetry parameter, $\hat{\alpha}$, differs from some value $\alpha_{0}$ using the results that $\sqrt{T}\left(\hat{\alpha}-\alpha_{0}\right) \rightarrow \mathcal{N}\left(0,\left(\hat{h}^{\prime} \hat{S}^{-1} \hat{h}\right)^{-1}\right)$, where $\hat{h}=\frac{1}{T} \sum_{t=\tau}^{T+\tau-1} v_{t} \mid \pi_{t+1}-$ $\left.\hat{\pi}_{t \mid t+1}\right|^{p-1}$. 
Equipped with the the estimated asymmetry parameter, $\hat{\alpha}$, we use a $J$-test for rationality of forecasts. Elliott et al. (2005) show that the $J$-test can be implemented as $J(\hat{\alpha})=\frac{1}{T}\left(x_{t}^{\prime} \hat{S}^{-1} x_{t}\right) \sim$ $\chi_{d-1}^{2}$, where $d>1, x_{t}=\sum_{t=\tau}^{T+\tau-1} v_{t}\left(I_{t+1}-\hat{\alpha}\right)\left|\pi_{t+1}-\hat{\pi}_{t \mid t+1}\right|^{p-1}$, and $d$ denotes the number of instruments. For a symmetric loss function, we have $J(0.5) \sim \chi_{d}^{2}$. A comparison of $J(\hat{\alpha})$ with $J(0.5)$ addresses the question of whether evidence against forecast rationality changes once we switch from an assumed symmetric to an estimated asymmetric loss function.

In the special case that $v_{t}$ is a constant and $p=1$, Equation (2) implies that the optimal estimate of the asymmetry parameter, $\hat{\alpha}$, is equal to the proportion of negative forecast errors. Whether it is optimal for a forecaster to produce many or few negative forecast errors depends on the shape of the potentially asymmetric loss function. Irrespective of the shape of the loss function, however, rationality of forecasts implies that it should not be possible to predict the sign of forecast errors with information in a forecasters' information set. A forecasters' information set comprises, among other information, the forecast itself. Hence, Patton and Timmermann (2007) propose to test for forecast optimality by regressing $I_{t+1}$ on the following "quantile model":

$$
I_{t+1}=\beta_{0}+\beta_{1} \hat{\pi}_{t \mid t+1}+u_{t+1},
$$

where $\beta_{0}$ and $\beta_{1}$ denotes the coefficients to be estimated, and $u_{t}$ denotes a stochastic disturbance term. Equation (3) can be estimated by the ordinary-least squares technique or, because of the dichotomous nature of the regressand, by a Probit / Logit model. Unlike the approach proposed by Elliott et al. (2005), Equation (3) does not require knowledge of the functional form of forecasters' loss function. Rather, Patton and Timmermann (2007) show that Equation (3) can be applied as long as forecasters' loss function is homogenous in the forecast error in case the inflation rate has dynamics in the conditional mean and the conditional variance. The mild technical requirement of homogeneity implies that the loss function can be of a very general, unknown form.

\section{Empirical Analysis}

\subsection{Micro-Level Inflation Forecasts}

Monthly survey data on forecasts of the inflation rate in South Africa were taken from Bloomberg. The forecasts are available at the micro-level of individual forecasters. The forecasts are shortterm forecasts because, for example, in May they predict the inflation rate released in June. The forecasts cover both the year-on-year CPI inflation rate and the year-on-year CPIX inflation rate, 
where the latter excludes mortgage costs. The forecasts of the CPI inflation rate are available for the sample period 2000/06-2014/07. The forecasts for the CPIX inflation rate are available for the sample period 2000/06-2009/01, because in 2009 the South African Reserve Bank switched from targeting the CPIX inflation rate to targeting the CPI inflation rate.

- Please include Figure 1 about here. -

Figure 1 plots summary statistics of the data. The panels on the left-hand-side show the inflation rates along with vertical bars that visualize the range of forecasts. The vertical bars illustrate that, while the forecasts track the paths of the inflation rates, cross-sectional heterogeneity of forecasts is a characteristic feature of the data. Furthermore, the cross-sectional heterogeneity of forecasts was not constant over time. The graphs also plot two dashed horizontal lines that indicate the official 3\% - 6\% inflation-targeting band of the South African Reserve Bank. The inflation rates climbed above the upper boundary of the inflation-targeting band by several percentage points in $2002 / 2003$, following a currency crisis in autumn/winter 2001, and $2007 / 2008$, due to a spike in food and energy prices.

The center panels of Figure 1 illustrate that the forecasts are available as an unbalanced panel because not all forecasters participated in all surveys. The minimum number of forecasts per forecaster is one, while the maximum is 161 for the CPI inflation rate and 98 for the CPIX inflation rate. Furthermore, as the panels on the right-hand side of Figure 1 indicate, the number of forecasts per survey increased over time in a non-monotonic way. In total, the survey data feature 2,691 forecasts of the CPI inflation rate and 1,418 forecasts of the CPIX inflation rate. In our empirical analysis, however, we do not use all forecasts but rather concentrate on the forecasts of those forecasters who contributed at least 25 forecasts, which is the case for 37 CPI forecasters and 24 CPIX forecasters.

Figure 2 summarizes the estimates of the asymmetry parameter, $\hat{\alpha}$, along with the results of the $J$-tests for forecast rationality for the case of a lin-lin loss function (for similar figures, see Fritsche et al. 2014). Figure 3 summarizes the estimation results for a quad-quad loss function. We report four models which differ in terms of the instruments included in order to test for robustness. Model 1 only includes a constant as an instrument; Model 2 includes a constant and lagged inflation; Model 3 includes a constant and the lagged forecast error; and, Model 4 includes a constant, lagged inflation, and the lagged forecast error (see also Elliott et al. 2005).

- Please include Figures 2 and 3 about here. - 
The general message conveyed by the box plots on the left-hand-side of Figure 2 and 3 is that, there is evidence of asymmetry of the loss function for some forecasters but not for others. Hence, there is cross-sectional heterogeneity with respect to the estimated asymmetry of the loss function. This heterogeneity tends to be larger for the forecasts of the CPIX inflation rate than for the CPI inflation rate, and for a quad-quad loss function than for a lin-lin loss function. For a lin-lin loss function, the estimates of the asymmetry parameter, $\hat{\alpha}$, tend to be smaller than the benchmark value of 0.5 . For the majority of forecasters, however, a test of the null hypothesis $\hat{\alpha}=0.5$ yields insignificant results, as shown in the scatter plots of the corresponding $p$-values in the center panels.

The scatter plots of the $p$-values for the corresponding $J$-tests appear on the right hand side of Figure 2 and 3. These indicate that, depending on the model being studied (instruments), the null hypothesis of rationality of forecasts cannot be rejected (at the $10 \%$ level of significance) for the majority of forecasters, irrespective of the symmetry or asymmetry of the loss function (i.e., $p(J(0.5))>0.1$ and $p(J(\hat{\alpha}))>0.1)$. We also observe that there are a few forecasters for which rationality can be rejected under a symmetric loss function, but not under an estimated asymmetric loss function $(p(J(0.5))<0.1$ and $p(J(\hat{\alpha}))>0.1)$. For only a few forecasters do the $J$-test reject forecast rationality irrespective of the assumed loss function $(p(J(0.5))<0.1$ and $p(J(\hat{\alpha}))<0.1)$, or strengthen the evidence against forecast rationality under an estimated asymmetric loss function $(p(J(0.5))>0.1$ and $p(J(\hat{\alpha}))<0.1)$.

On balance, our results show that their is some heterogeneity across forecasters with respect to the shape of the loss function and the rationality of forecasts. This is not surprising as forecasters are from different industries, where they face different incentives to forecast strategically. In some industries forecast accuracy may be highly valued, whereas in some other industries publicity may be important, which could be an incentive for forecasters to try and stand out from the crowd by forecasting away from the consensus forecast. Different incentives, in turn, are likely to result in different shapes of the loss functions and different results for the rationality test. Forecasters may find it fully rational to form a strategic forecast given some non-standard, asymmetric loss function. If so, testing for rationality of forecasting by invoking an assumed symmetric loss function will produce strong evidence against forecast rationality. Conversely, even if we find evidence against forecast rationality for some forecasters under an estimated asymmetric loss function, we cannot definitively conclude that forecasts are not rational. Rather, any test of forecast rationality is subject to the well-known joint-hypothesis problem, and the tests we have studied in this research are no exception. The joint-hypothesis problem stipulates that a test of the rationality of forecasts, by construction, is always also a test of an assumed economic or statistical model. It is, therefore, important to study forecast rationality using an alternative empirical method. 
- Please include Tables 1 and 2 about here. -

Hence, we next study estimation results for Equation (3). Tables 1 and 2 summarize the results. The estimation results for the "quantile model" corroborate the results of the $J$-tests. Most estimates of the coefficients of the "quantile model" are insignificant, consistent with forecast rationality.

Finally, it is worth mentioning that the signs of the slope coefficient, $\hat{\beta}_{1}$, of the "quantile model" switch between being positive and being negative, and there is no apparent recognizable pattern. The unsystematic changing of the sign of the slope coefficient is consistent with findings recently reported by Pierdzioch et al. (2014b), who find signs of time-varying forecaster herding in South African inflation expectations. According to their findings, forecaster herding tends to be strong when inflation volatility is high. In contrast, forecasters appear to anti-herd in times of low inflation volatility. Forecaster herding suggests that forecasters tend to group their forecasts closely around a consensus forecast, implying that the chance of observing a negative (positive) forecast error (actual inflation rate - forecast) decreases relative to the case of unbiased forecasts. Hence, as studied in depth by Fritsche et al. (2014), forecaster herding should result, in terms of the "quantile model", in a positive slope coefficient, $\hat{\beta}_{1}$, because a large forecast reduces the probability of observing $I_{t+1}=1$. In contrast, forecaster anti-herding should result in a negative slope coefficient. As a consequence, if forecaster herding varies over time, one would expect a mixture of positive and negative slope coefficients, most of which are insignificant. This is exactly the pattern that we observe in our data.

\subsection{Pooled and Sectoral Inflation Forecasts}

As another exercise, we pool the Bloomberg data across forecasters, and we compare the results with results for the BER data, which have been studied widely in earlier literature. The BER data are available at a quarterly frequency for the sample period 2000Q2-2014Q2. The sample period is constrained by the fact that the BER began surveying inflation expectations in 2000Q2 and a quarterly frequency is used to align with the frequency at which the survey is conducted. The final dataset consists of inflation, inflation expectations, inflation forecast errors and lagged versions of some of these variables, resulting in a sample period of 2000Q3-2014Q2 for this study. The BER data is a survey of forecasters representing three groups within South Africa: the financial sector, the business sector, and trade unions. The survey is conducted on behalf of the South African Reserve Bank. According to the detailed description of the BER data available on the web page of the Bureau for Economic Research (2000-2014), the panel of forecasters participating in the 
BER questionnaires consists of 312 business executives, 17 financial analysts, and 34 forecasters from trade unions. Thus, the number of respondents surveyed each quarter is documented. The numbers may change over time and we cannot tell if they are the same participants over time. The individual level forecasts, however, are not published. Only the sectoral data are publicly available.

There are a number of other features of this survey that need to be highlighted. First, the respondents forecast the CPIX (the consumer price index excluding mortgage rates) from 2000Q3 to 2008Q4 and the CPI (the headline consumer price index) from 2009Q1 to 2014Q2, as a result of a change in 2009 of the proxy for inflation that the the South African Reserve Bank officially targeted. In this study, the focus is on the forecasting of inflation itself, and the series we use as a proxy for inflation consists of the CPIX up to the end of 2008 and the CPI thereafter, which we call the 'targeted inflation index'. Our targeted inflation index captures the measure of inflation officially targeted and communicated by the South African Reserve Bank. As a consequence of the change in the officially targeted inflation rate, the BER surveyed the expectations of the CPIX up to the end of 2008 and only the CPI thereafter. In line with Reid (2012), we assume that both the targeted price index and expectations thereof are continuous time-series. Figure 4 supports this assumption as there was a smooth transition between the data series in 2008/2009.

- Please include Figure 4 about here. -

Second, the original survey data consists of calendar year forecasts rather than forecasts with a constant one year horizon. Each quarter, survey respondents were asked to forecast inflation for the current and following two calendar years (e.g., in 2000Q1 they were asked to forecast what inflation would be at the end of 2000, at the end of 2001 and at the end of 2002). Crucially, in Q1 respondents forecast a larger proportion of the current year than in the later quarters of the year. Therefore, the horizons of the predictions change from quarter to quarter. In order to be able to analyze inflation expectations with a constant forecast horizon, we construct a time series of inflation expectations with a 1-year horizon from the original survey data. Accordingly, to account for the quarter of the year in which the survey is being conducted, in each quarter we add a fraction of the current year to a fraction of the following year. To give an example, the 2010Q4 survey (for which the field work took place in October) asked forecasters to predict what CPI would be at the end of 2010 and at the end of 2011. Hence, we constructed the one-year forecast by combining $25 \%$ of the current year's forecast with $75 \%$ of the following year's forecast.

Figure 4 illustrates the resulting constant one-year horizon forecasts. The actual inflation series plotted in Figure 4 is a construction of the average 'real time targeted inflation' over the past 
twelve months. This data is based on two inflation series collected by Statistics South Africa, but it has been constructed to capture some particular characteristics. Firstly, it is a real time dataset constructed from the original statistical releases archived on the Statistics South Africa website. Secondly, this inflation series consists of CPIX from 2000Q2 to 2008Q4 and CPI thereafter because, following an announcement by the Minister of Finance in October 2008, the South African Reserve Bank began, in January 2009, to use the CPI rather than the CPIX as its official proxy for inflation, as discussed above. It is in this sense that we refer to this variable as the 'targeted inflation index'. We argue that this is a reasonable approach to take, given that the focus in this study is on inflation expectations. One of the primary aims of inflation targeting is to anchor inflation expectations to the announced target, so theoretically the public in South Africa should simply have changed from anchoring their expectations on the CPIX to the CPI if they believe that is what the South African Reserve Bank is targeting (assuming an acceptable degree of credibility of monetary policy).

Figure 4 also shows the proportion of negative forecast errors for the three groups of forecasters, as Equation (2) implies that this proportion determines the optimal estimate of the asymmetry parameter, $\hat{\alpha}$, when $v_{t}$ is a constant and $p=1$. We have $44 \%$ negative forecast errors for financial analysts, and $66 \&$ and $68 \%$ for the business sector and trade unions when evaluated for the full sample of data. Hence, we expect to find that the loss from overestimation of the inflation rate is on average smaller than the loss from underestimation of similar magnitude, but also that the implied asymmetry of the loss function is less pronounced in case of financial analysts than in the case of the other two groups of forecasters. In economic terms, the finding that price setters (trade unions and the business sector) experience a higher loss when underestimating rather than overestimating the inflation rate is not surprising. Figure 4 illustrates this finding by plotting lin-lin loss functions for the three groups of forecasters with an asymmetry parameter equal to the proportion of forecast errors. While the loss function of financial analysts is relatively (but not fully) symmetric, the loss functions for the other two groups of forecasters give rise to a larger loss in case of an underestimation than in case of an overestimation. As a result, the inflation expectations of price setters should on average be higher than the inflation expectations of financial analysts. More specifically, Figure 4 shows that all three groups of BER forecasters tend to underestimate the inflation rate during periods of rising inflation, while during disinflationary episodes, the inflation expectations of price setters tend to return in a much slower fashion to lower levels than the inflation expectations of financial analysts. The sluggish return of inflation expectations of price setters to lower levels after periods of high inflation illustrates why Kabundi and Schaling (2014) might have reached the conclusion that the inflation expectations of price setters are less well anchored than the inflation expectations of financial analysts.

Table 3 summarizes the estimation results. Panel A shows the results for the asymmetry parameter, 
and Panel B shows the results of the rationality tests. We report results both for the pooled Bloomberg data and the sectoral BER data. As for the estimated asymmetry coefficient, the results for the Bloomberg data corroborate the findings summarized in Figures 2 and 3. The asymmetry parameter is smaller than its benchmark value, with the asymmetry parameter estimated on the pooled CPIX data somewhat smaller than the asymmetry parameter estimated on the pooled CPI data. The standard error in the case of the pooled CPIX data is larger than that of the pooled CPI data. Estimates are highly stable across the four different models being studied. Importantly, the $J$-tests are all highly significant. Hence, pooling the forecasts implies that evidence against forecast rationality mounts despite the result that many of the rationality tests estimated on individual forecasts yield insignificant results. This noticeable difference between the results for individual and pooled forecasts is not surprising given that Figures 2 and 3 highlight a certain degree of cross-sectional heterogeneity in the loss functions. In other words, pooling heterogeneous forecasts leads to an "aggregation bias", to the extent that the pooled data leads us to conclude erroneously that forecasts violate the rationality hypothesis.

- Please include Table 3 about here. -

Turning next to the results for the BER data, the estimates of the asymmetry parameter in the case of financial analysts is close to the estimate computed for the Bloomberg data under Models 1 and 2. For both models, the asymmetry parameter is not different from 0.5 - the value it assumes under a symmetric loss function. With regard to the larger standard error we observe for the BER results, it should be noted, however, that we have only 50 observations after removing missing data, while we can use 1,914 observations in case of the Bloomberg data. It is, therefore, not surprising that also the variability of the estimates of the asymmetry parameter is substantial across the estimated models for the BER data. As the analysis of Figure 4 already made clear, the estimates of the asymmetry parameter are larger for the business sector and trade unions than for financial analysts. Also in line with the message conveyed by Figure 4, is the result that the forecasts of the business sector and the trade unions closely track each other, while both series differ to a non-negligible extent from the forecasts of financial analysts (see Ehlers and Steinbach 2007 and Kabundi and Schaling 2014). While the interpretation of the results should not be stretched too far given the limited number of observations, the comparatively large asymmetry parameter (which even increases when we move from Model 1 to Model 4) suggests that forecasts of price setters reflect that underestimation of the inflation rate is more costly than overestimation. Hence, as shown in Figure 4, forecasters will form forecasts such that the proportion of negative forecast errors (that is, the forecast exceeds the actual inflation rate) is larger than the proportion of positive forecast errors. 
Finally, the rationality tests are highly significant. Hence, as in the case of the Bloomberg data, the results for the sectoral BER data signal that violations of forecast rationality are prevalent among forecasters. However, as we know from our study of the Bloomberg data, estimation results for pooled sectoral data can be quite misleading if individual forecasters are heterogeneous with regard to the shapes of their loss functions and their overall forecasting behavior. Hence, the interpretation of the results of the rationality tests for the sectoral BER data should not be stretched too far. Rather, the results of our analysis suggest that a deeper understanding of the properties of the forecasts by the business sector and trade unions requires access to the disaggregated data.

\section{Concluding Remarks}

Using forecasts of the inflation rate in South Africa, we study the rationality of forecasts and the shape of forecasters' loss function. We find strong micro level evidence of forecast rationality. Evidence of the asymmetry of forecasters' loss function is mixed, indicating the presence of at least some extent of heterogeneity with respect to the shape of forecasters' loss functions. For a large proportion of forecasters, we cannot reject the hypothesis of forecast rationality. An estimated asymmetric loss function brings forecasts in some cases closer in line with forecast rationality, but the results depend on the model being studied. In contrast, we largely reject the hypothesis of forecast rationality when we study aggregated forecasts. We observe violations of forecast rationality for both the pooled Bloomberg data (which is a comparable survey focusing only on financial analysts) and the sectoral BER data. A comparison with our results for individual forecasts, however, shows that the deviations from rationality in the case of pooled forecasts and sectoral forecasts should be interpreted with caution as forecaster heterogeneity at the microeconomic level of individual forecasters can lead to spurious deviations from forecast rationality at the aggregated level of pooled and sectoral data. Hence, the main message to take home from our our empirical analysis is that, given that the concept of forecast rationality is of key importance for monetary policy, it is advantageous to assess the rationality of South African inflation expectations, and the functional form of forecaster's loss function, using micro-level data.

\section{References}

Bureau for Economic Research (2000-2014). Inflation expectations survey (each quarter between 2000Q2 and 2014Q2). Available at: http://www.ber.ac.za/surveys/2002.aspx? documenttype=Inflation\%20Exectations 
Christodoulakis, G.A., and E.C. Mamatzakis (2008). Assessing the prudence of economic forecasts in the EU. Journal of Applied Econometrics 24: 583-606.

Döpke, J., U. Fritsche, and B. Siliverstovs (2010). Evaluating German business cycle forecasts under an asymmetric loss function. Journal of Business Cycle Measurement and Analysis 2010/1: $1-18$.

Ehlers, N., M. R. Steinbach (2007). The formation of inflation expectations in South Africa. Working Paper WP/07/06, South African Reserve Bank.

Elliott, G., I. Komunjer, and A. Timmermann (2005). Estimation and testing of forecast rationality under flexible loss. Review of Economics Studies 72: 1107-1125.

Elliott, G., Komunjer, I. and A. Timmermann (2008). Biases in macroeconomic forecasts: irrationality or asymmetric loss? Journal of the European Economic Association 6: 122-57.

Figlewski, S., and P. Wachtel (1981). The formation of inflationary expectations. Review of Economics and Statistics 63: 1-10.

Fritsche, U., C. Pierdzioch, J.C. Rülke, and G. Stadtmann (2014). Forecasting the Brazilian real and the Mexican peso: Asymmetric loss, forecast rationality, and forecaster herding. International Journal of Forecasting (forthcoming).

Kabundi, A., E. Schaling (2013). Inflation and inflation expectations in South Africa: An attempt at explanation. South African Journal of Economics 81: 346-355.

Kabundi, A., Schaling, E., M. Some (2014). Monetary policy and heterogeneous inflation expectations in South Africa. Working Paper No. 422, Economic Research Southern Africa.

Keane, M.P., and D.E. Runkle (1990). Testing the rationality of price forecasts: New evidence from panel data. American Economic Review 80: 714-735.

Kose, M.A., and E.S. Prasad (2010). Emerging markets: Resilience and growth amid global turmoil. Washington D.C: The Brookings Institution Press.

Laster D., Bennett, P., S. Geoum (1999). Rational Bias in Macroeconomic Forecasts. Quarterly Journal of Economics 114: 293-318.

Nunes, R. (2010). Inflation dynamics: The role of expectations. Journal of Money, Credit, and Banking 42: 1161-1172.

Patton, A. J., and A. Timmermann (2007). Testing forecast optimality under unknown loss. Journal of the American Statistical Association 102: 1172-1184.

Pierdzioch, C., J.C. Rülke, P. Tillmann (2014a). Using Forecasts to Uncover the Loss Function of FOMC Members. Macroeconomic Dynamics (forthcoming).

Pierdzioch, C., Reid, M. B., R. Gupta (2014b). Inflation forecasts and forecaster herding: Evidence from South African survey data. Department of Economics, University of Pretoria, Working Paper No. 2014-55.

R Development Core Team (2014). R: A language and environment for statistical computing. Internet address: http://www.R-project.org. 
Reid, M. B. (2012). Inflation expectations of the inattentive general public. Working Paper No. 278, Economic Research Southern Africa.

Reid, M.B., and S. du Plessis (2011). Talking to the inattentive public: How the media translates the Reserve Bank's communications. Working Paper No. 254, Economic Research Southern Africa.

Rich, R.W. (1989). Testing the rationality of inflation forecasts from survey data: Another look at the SRC expected price change data. Review of Economics Studies 71: 682-686.

Roberts, J.M. (1997). Is inflation sticky? Journal of Monetary Economics 39: 173-196. 
Figure 1: Summary Statistics of the Bloomberg Data
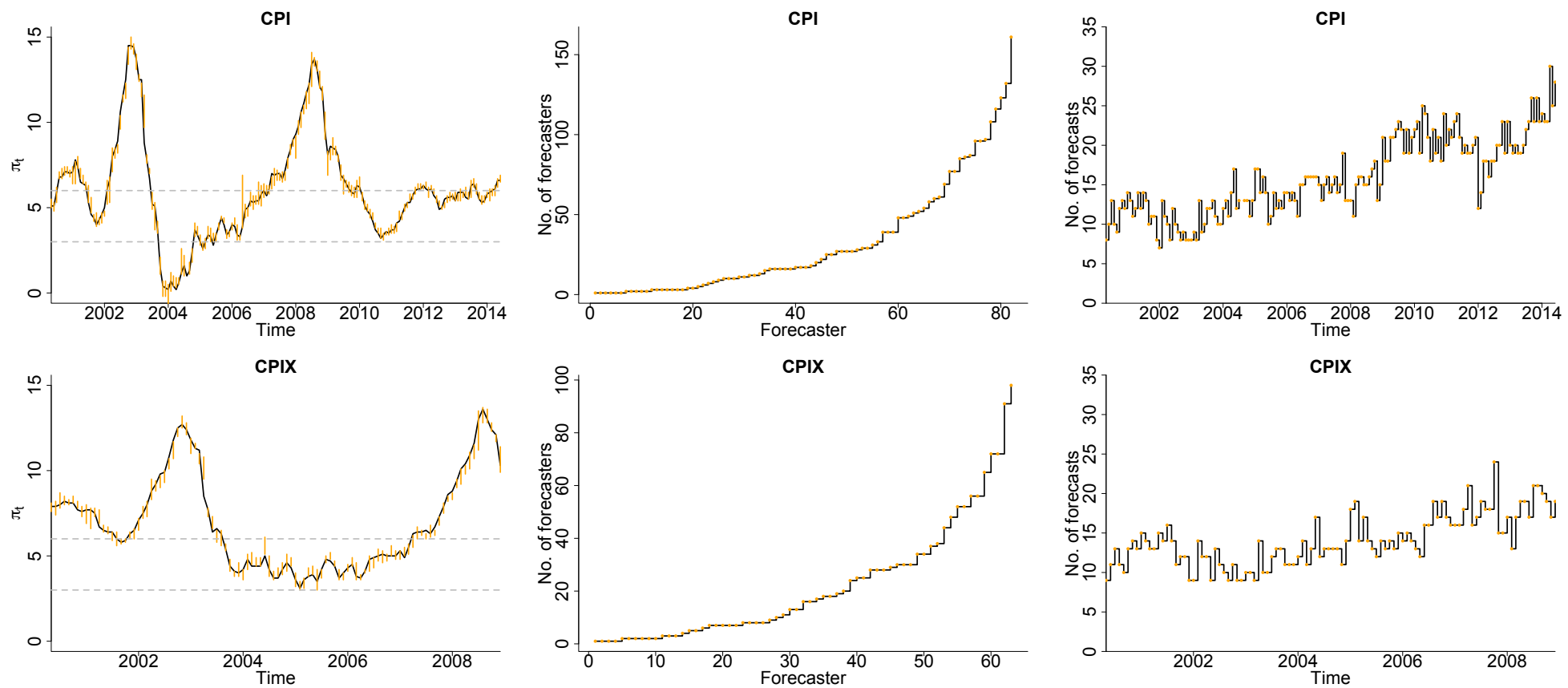

Note: $\pi_{t+1}$ actual inflation rate. Vertical bars $=$ cross sectional range of forecasts. This figure and all other figures and results were computed using the free programming environment R (R Development Core Team 2014). 
Figure 2: Estimation Results (Lin-Lin Loss Function)
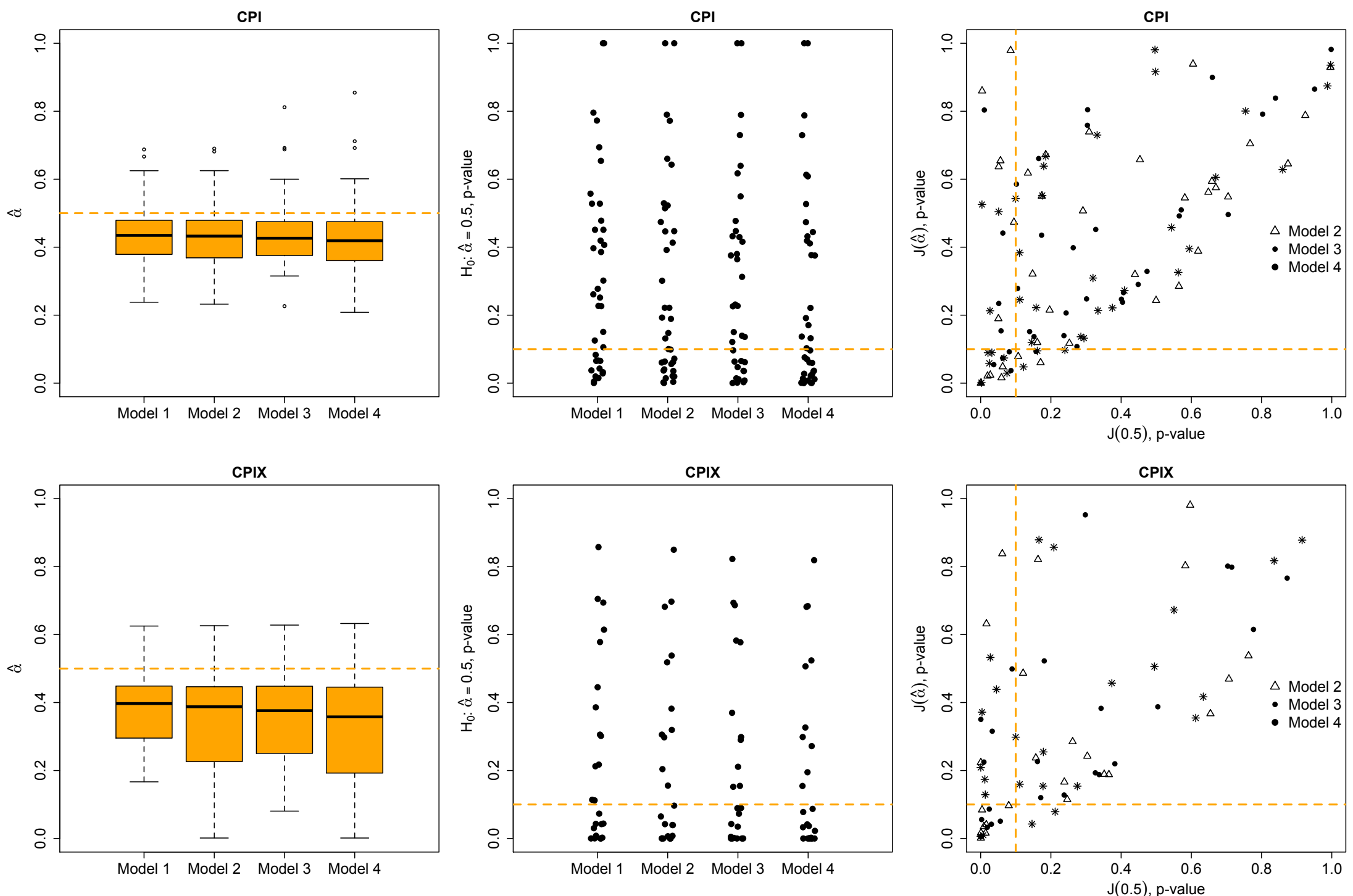

Note: The instruments being used are: a constant (Model 1), a constant and the lagged inflation rate (Model 2), a constant and the lagged forecast error (Model 3), and a constant, the lagged inflation rate, and the lagged forecast error (Model 4). The dashed horizontal line in the graphs in the middle and on the right-hand side denote a significance level of $10 \%$. 
Figure 3: Estimation Results (Quad-Quad Loss Function)

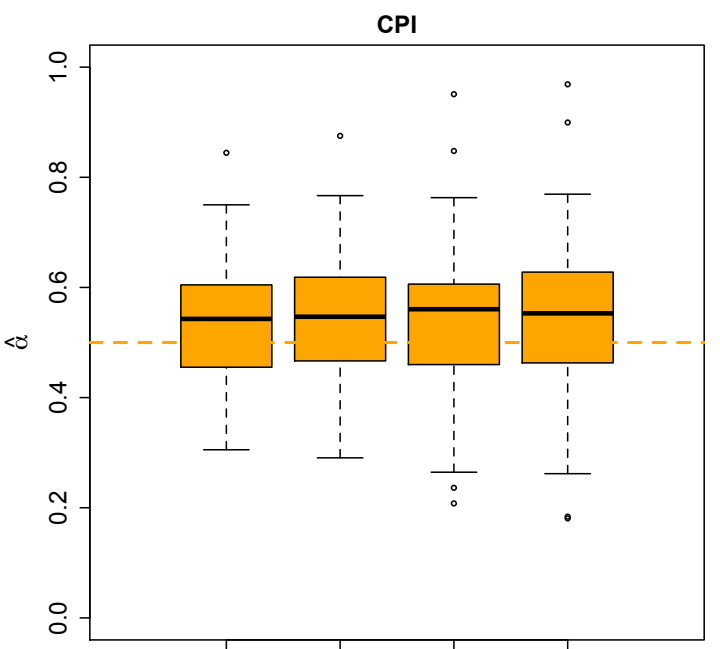

Model 1 Model 2 Model $3 \quad$ Model 4

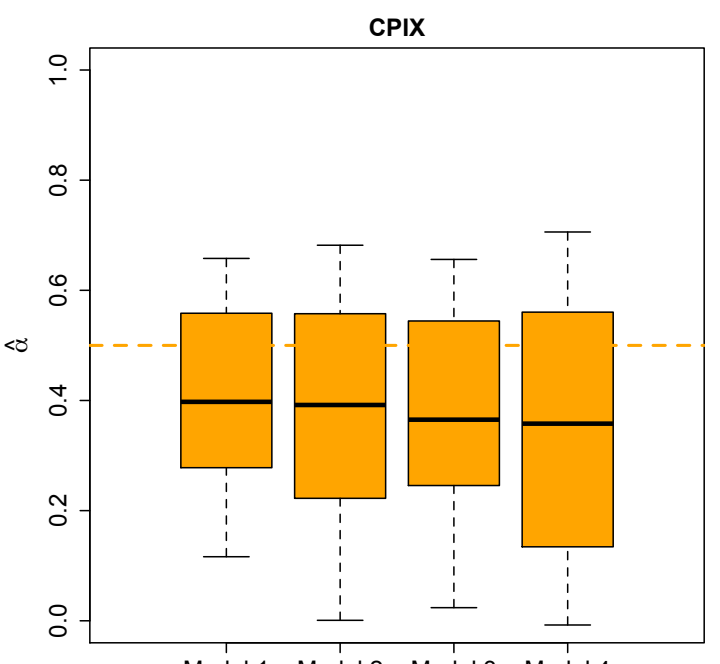

CPI

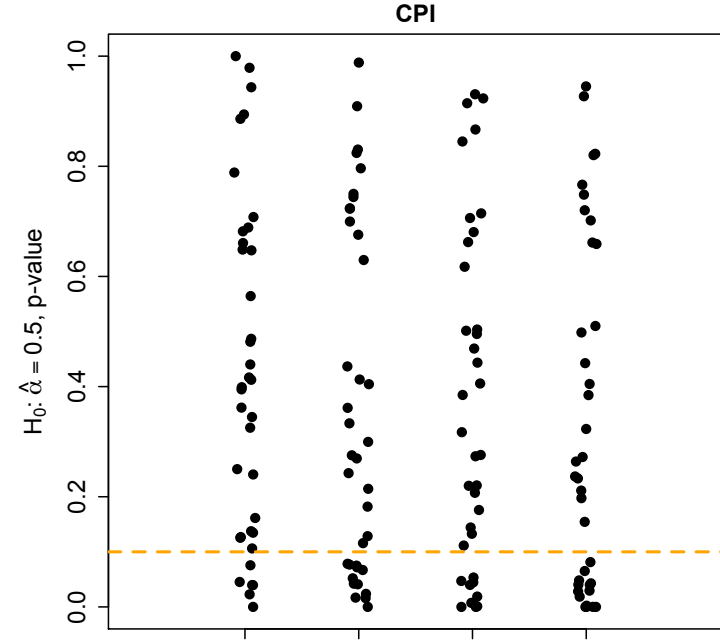

Model 1 Model 2 Model 3 Model 4

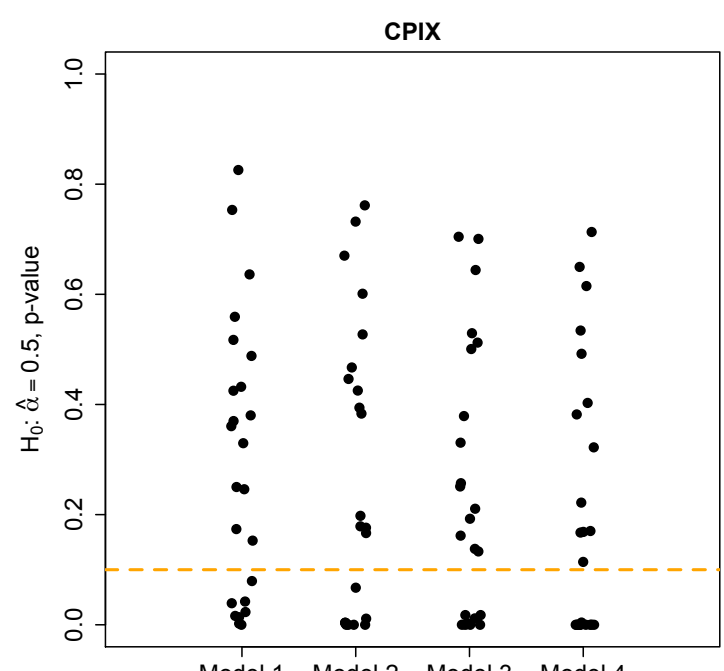

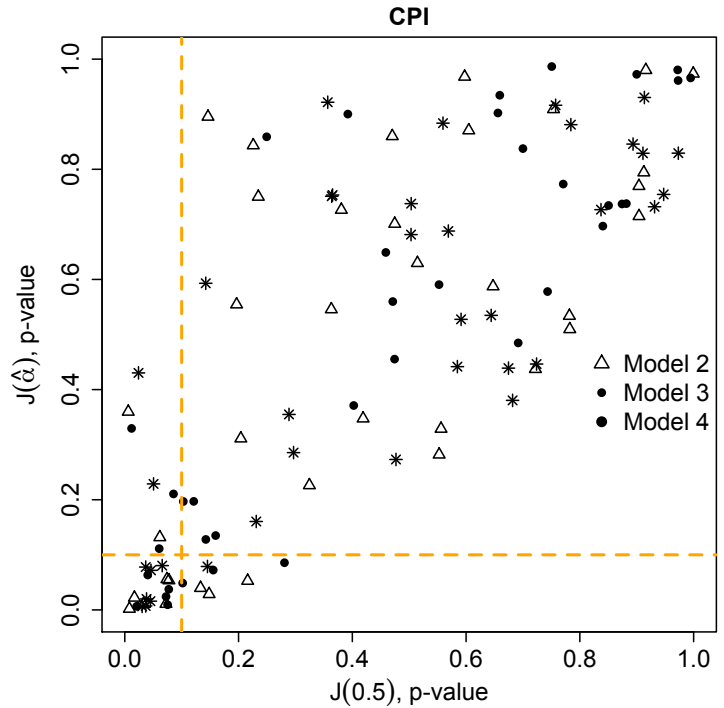

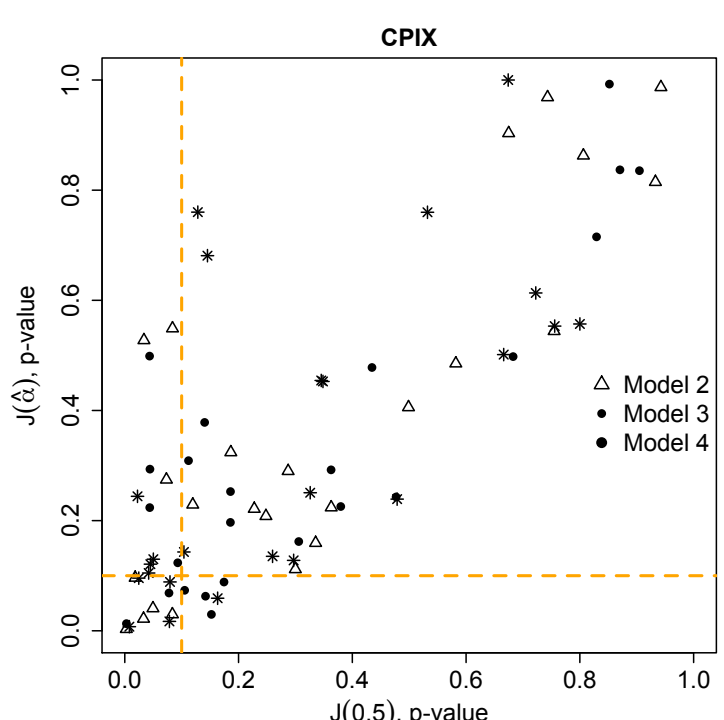

Note: The instruments being used are: a constant (Model 1), a constant and the lagged inflation rate (Model 2), a constant and the lagged forecast error (Model 3), and a constant, the lagged inflation rate, and the lagged forecast error (Model 4). The dashed horizontal line in the graphs in the middle and on the right-hand side denote a significance level of $10 \%$. 
Figure 4: Summary Statistics of the BER Data

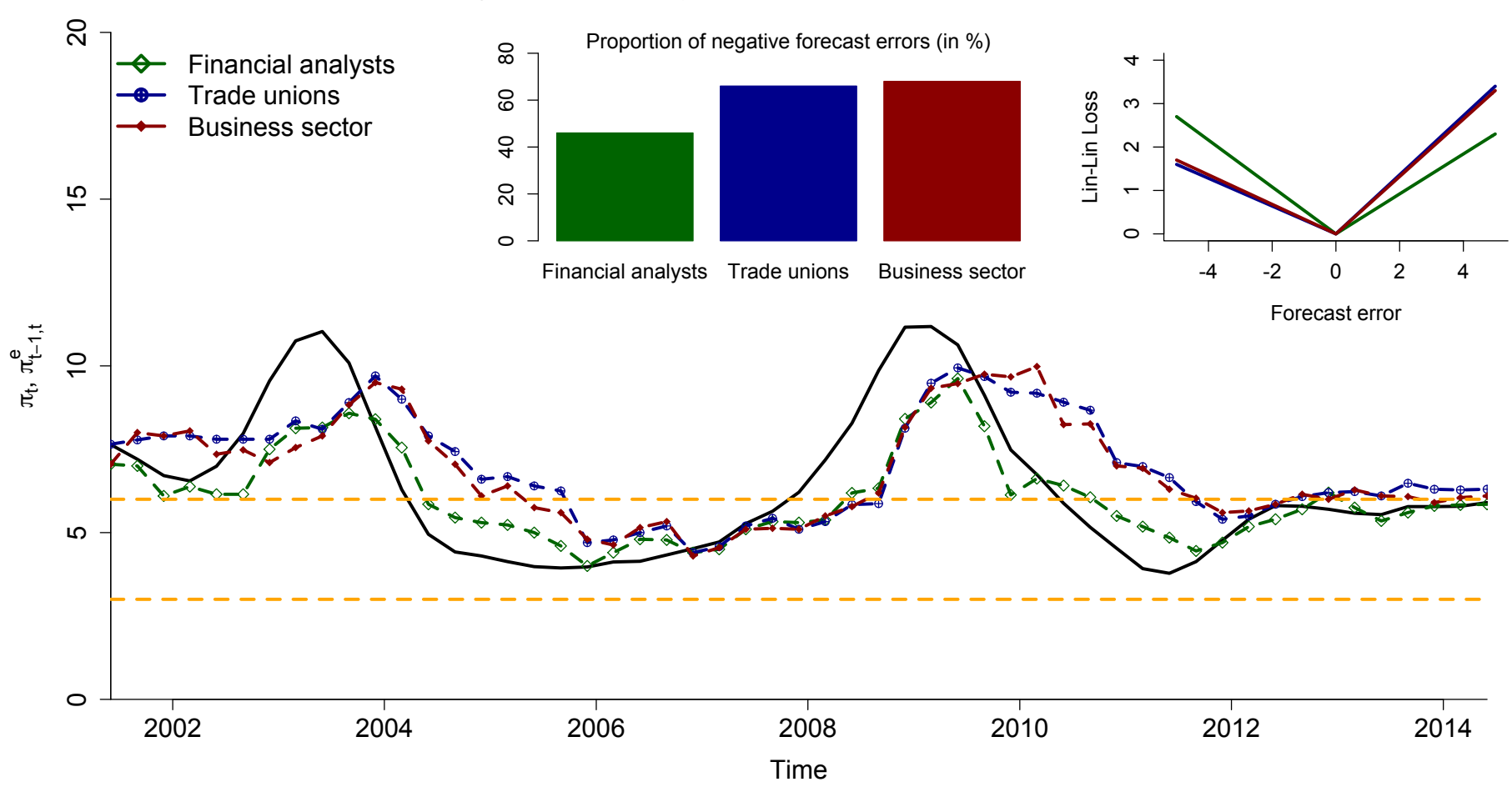

Note: The forecast error is defined as the actual inflation rate minus the forecast of the inflation rate. The forecast error is defined as the actual inflation rate minus the forecast of the inflation rate. As for the lin-lin loss functions shown in the upper-right plot, it should be noted that the proportion of negative forecast errors varies between $66 \%$ and $68 \%$ for the business sector and trade unions. As a result, the loss functions of these two groups of forecasters more or less coincide. 
Table 1: Logit Estimates of the Quantile Model (CPI)

\begin{tabular}{lrrrr}
\hline \hline Obs. & \multicolumn{1}{c}{$\hat{\beta}_{0}$} & p-value & \multicolumn{1}{c}{$\hat{\beta_{1}}$} & p-value \\
\hline 22 & 2.56 & 0.16 & -0.54 & 0.11 \\
53 & 0.76 & 0.64 & -0.22 & 0.43 \\
41 & 1.88 & 0.12 & -0.45 & 0.04 \\
48 & 0.36 & 0.56 & -0.07 & 0.41 \\
16 & -0.31 & 0.86 & 0.23 & 0.64 \\
73 & 1.27 & 0.15 & -0.23 & 0.10 \\
86 & 2.32 & 0.01 & -0.43 & 0.00 \\
21 & 1.88 & 0.10 & -0.33 & 0.03 \\
64 & 0.38 & 0.61 & -0.03 & 0.78 \\
37 & -5.97 & 0.04 & 1.03 & 0.05 \\
45 & 0.50 & 0.58 & -0.09 & 0.47 \\
23 & -22.38 & 0.06 & 3.73 & 0.07 \\
35 & -3.25 & 0.20 & 0.66 & 0.15 \\
16 & 0.83 & 0.31 & -0.01 & 0.95 \\
52 & -0.84 & 0.29 & 0.04 & 0.78 \\
69 & -0.02 & 0.97 & -0.03 & 0.74 \\
15 & 4.67 & 0.60 & -0.82 & 0.59 \\
95 & -0.25 & 0.60 & -0.04 & 0.58 \\
72 & 0.33 & 0.52 & -0.03 & 0.72 \\
53 & 0.31 & 0.84 & -0.15 & 0.59 \\
82 & -0.94 & 0.12 & 0.03 & 0.75 \\
64 & 0.22 & 0.86 & -0.07 & 0.74 \\
24 & 1.20 & 0.30 & -0.40 & 0.26 \\
154 & 0.18 & 0.63 & -0.05 & 0.37 \\
89 & 1.18 & 0.15 & -0.25 & 0.05 \\
116 & -0.75 & 0.08 & 0.06 & 0.34 \\
26 & -6.20 & 0.33 & 0.99 & 0.36 \\
45 & -0.23 & 0.76 & -0.05 & 0.59 \\
26 & 1.48 & 0.34 & -0.24 & 0.28 \\
50 & -2.62 & 0.12 & 0.55 & 0.08 \\
21 & -1.39 & 0.27 & 0.03 & 0.84 \\
111 & 0.01 & 0.97 & -0.05 & 0.45 \\
28 & -0.21 & 0.84 & 0.03 & 0.83 \\
23 & 0.25 & 0.90 & -0.09 & 0.79 \\
24 & 2.35 & 0.18 & -0.30 & 0.32 \\
23 & -1.82 & 0.35 & 0.28 & 0.41 \\
72 & -0.09 & 0.87 & -0.08 & 0.41 \\
\hline \hline & & & & \\
& & &
\end{tabular}

Note: Empirical model: $I_{t+1}=\beta_{0}+\beta_{1} \hat{\pi}_{t \mid t+1}+u_{t}$, estimated by means of a logit model. Obs. $=$ Number of usable observations. This number can be smaller than 25 after removing missing values. 
Table 2: Logit Estimates of the Quantile Model (CPIX)

\begin{tabular}{lrrrr}
\hline \hline Obs. & \multicolumn{1}{c}{$\hat{\beta}_{0}$} & p-value & \multicolumn{1}{c}{$\hat{\beta}_{1}$} & p-value \\
\hline 26 & -0.54 & 0.67 & -0.06 & 0.70 \\
41 & 1.65 & 0.25 & -0.50 & 0.06 \\
52 & 1.31 & 0.17 & -0.20 & 0.11 \\
16 & -1.24 & 0.81 & 0.40 & 0.73 \\
33 & 10.64 & 0.02 & -1.97 & 0.02 \\
22 & 1.38 & 0.30 & -0.22 & 0.18 \\
28 & 0.65 & 0.56 & -0.11 & 0.46 \\
28 & -0.42 & 0.73 & -0.04 & 0.77 \\
16 & 0.94 & 0.69 & -0.13 & 0.77 \\
33 & 0.43 & 0.70 & -0.17 & 0.43 \\
31 & 0.62 & 0.44 & -0.10 & 0.34 \\
52 & -0.98 & 0.25 & 0.05 & 0.59 \\
60 & 0.18 & 0.78 & -0.10 & 0.34 \\
33 & -3.08 & 0.01 & 0.23 & 0.08 \\
26 & -6.97 & 0.07 & 1.71 & 0.06 \\
93 & 1.07 & 0.09 & -0.21 & 0.02 \\
28 & 2.69 & 0.24 & -0.48 & 0.11 \\
62 & -0.40 & 0.52 & 0.02 & 0.80 \\
42 & 1.21 & 0.35 & -0.22 & 0.19 \\
28 & 2.19 & 0.36 & -0.32 & 0.30 \\
21 & -2.48 & 0.23 & 0.11 & 0.60 \\
81 & 0.29 & 0.65 & -0.08 & 0.35 \\
46 & 0.97 & 0.39 & -0.30 & 0.15 \\
\hline \hline
\end{tabular}

Note: Empirical model: $I_{t+1}=\beta_{0}+\beta_{1} \hat{\pi}_{t \mid t+1}+u_{t}$, estimated by means of a logit model. Obs. $=$ Number of usable observations. This number can be smaller than 25 after removing missing values. 
Table 3: Pooled Data

Panel A: Estimated Asymmetry Parameter

\begin{tabular}{lcrrrrrrrrrrr}
\hline \hline \multicolumn{1}{c}{ Data } & $\hat{\alpha}_{1}$ & $S E_{1}$ & $\mathrm{p}$-value & $\hat{\alpha}_{2}$ & $S E_{2}$ & $\mathrm{p}$-value & $\hat{\alpha}_{3}$ & $S E_{3}$ & $\mathrm{p}$-value & $\hat{\alpha}_{4}$ & $S E_{4}$ & $\mathrm{p}$-value \\
\hline Pooling (CPI data) & 0.44 & 0.01 & $<0.01$ & 0.44 & 0.01 & $<0.01$ & 0.44 & 0.01 & $<0.01$ & 0.44 & 0.01 & $<0.01$ \\
Pooling (CPIX data) & 0.38 & 0.02 & $<0.01$ & 0.38 & 0.02 & $<0.01$ & 0.38 & 0.02 & $<0.01$ & 0.38 & 0.02 & $<0.01$ \\
Financial analysts & 0.46 & 0.07 & 0.57 & 0.45 & 0.07 & 0.49 & 0.38 & 0.07 & 0.08 & 0.35 & 0.07 & 0.02 \\
Business sector & 0.66 & 0.07 & 0.02 & 0.72 & 0.06 & 0.00 & 0.84 & 0.05 & $<0.01$ & 0.87 & 0.05 & $<0.01$ \\
Trade unions & 0.68 & 0.07 & 0.01 & 0.75 & 0.06 & 0.00 & 0.87 & 0.05 & $<0.01$ & 0.90 & 0.04 & $<0.01$ \\
\hline \hline
\end{tabular}

Panel B: Rationality Tests

\begin{tabular}{|c|c|c|c|c|c|c|c|c|c|c|c|c|}
\hline Data & $J(0.5)_{2}$ & $\mathrm{p}$-value & $J(0.5)_{3}$ & p-value & $J(0.5)_{4}$ & p-value & $J(\hat{\alpha})_{2}$ & p-value & $J(\hat{\alpha})_{3}$ & $\mathrm{p}$-value & $J(\hat{\alpha})_{4}$ & p-value \\
\hline Pooling (CPI data) & 46.22 & $<0.01$ & 50.15 & $<0.01$ & 60.56 & $<0.01$ & 16.8 & $<0.01$ & 20.39 & $<0.01$ & 31.34 & $<0.01$ \\
\hline Pooling (CPIX data) & 73.09 & $<0.01$ & 54.03 & $<0.01$ & 73.64 & $<0.01$ & 23.7 & $<0.01$ & 4.48 & 0.03 & 24.14 & $<0.01$ \\
\hline Financial analysts & 4.74 & 0.09 & 15.86 & $<0.01$ & 19.00 & $<0.01$ & 4.52 & 0.03 & 16.57 & $<0.01$ & 18.50 & $<0.01$ \\
\hline Business sector & 13.35 & 0.00 & 25.24 & $<0.01$ & 25.84 & $<0.01$ & 6.67 & 0.01 & 13.20 & $<0.01$ & 14.19 & $<0.01$ \\
\hline Trade unions & 15.91 & 0.00 & 27.01 & $<0.01$ & 27.29 & $<0.01$ & 7.32 & 0.01 & 12.72 & $<0.01$ & 13.66 & $<0.01$ \\
\hline
\end{tabular}

Note: Results are for alin-lin loss function. The subindex denotes the model being estimated. The instruments being used are: a constant (Model 1), a constant and the lagged inflation rate (Model 2), a constant and the lagged forecast error (Model 3), and a constant, the lagged inflation rate, and the lagged forecast error (Model 4) 